\title{
Hydrated Nonpolar Solute Volumes: Interplay between Size, Attractiveness, and Molecular
} Structure

Henry S. Ashbaugh, ${ }^{*}$ J. Wesley Barnett, Natalia da Silva Moura, Hayden E. Houser

Department of Chemical and Biomolecular Engineering, Tulane University, New Orleans, LA 70118

A solute's partial molar volume determines its response to pressure, which can result in changes in molecular conformation or assembly state. Computing speed advanceshave made accurate partial molar volume evaluation in water routine, allowing for the dissection of themolecular factors underlying this significant thermodynamic variable. A recent simulation analysis of the volumes of nonpolar molecular solutes in waterreported that the apparent solventfree border thickness enshrouding these solutes grows with increasing solute size, based on the assumption the solute can be treated as an individual sphere [Biophys. Chem. 161 (2012) 46]. Thissuggests the solvent dewets these solutesas they grow in size. Via simulations of dewetted repulsive spherical solutes, we show that the border thicknesses ofthe largest non-polar molecular solutes tend towards that of a repulsive sphere. When attractive interactions are accounted for, however, the spherical solute border thicknessesfall below that of the largest molecular solutes. We demonstrate that if the molecular solutes are treated with atomic detail rather than approximated as anindividual sphere, the border thickness variationis minimal. Ageometric modelis put forward that reproduces theinferred border thickening, indicating the implied dewettingresults from a breakdown in the spherical volume approximation.

Under the assumption that the hydrated volume of a nonpolar solute can be approximately treated as the packing of water about a sphere, the phenomenological expression

$$
V_{\mathrm{hyd}} \approx 4 \pi\left(r_{\mathrm{vdW}}^{\mathrm{eff}}+\delta_{\mathrm{sph}}\right)^{3} / 3
$$

has proven useful to correlatepartial molar volume results[1-3]. The hydrated volume, $V_{\mathrm{hyd}}$, is determined as the solute's partial molar volume less that of an ideal gas particle in solution (i.e.,

\footnotetext{
* corresponding author: hanka@tulane.edu
} 
$V_{\text {hyd }}=\bar{V}_{\text {solute }}-R T \kappa_{0}$ where $R T$ is the product of the ideal gas constant and the temperature and $\kappa_{0}$ is the pure solvent compressibility). The effective van der Waals radius in eq. (1)is determined as $r_{\mathrm{vdW}}^{\text {eff }}=\left(3 V_{\mathrm{vdW}} / 4 \pi\right)^{1 / 3}$, where the solute's entire van der Waals volume $\left(V_{\mathrm{vdW}}\right)$ is collapsedonto a single sphere. The remaining term in this expression, $\delta_{\mathrm{sph}}$, is the solute's spherical border thickness, whichapproximates water packing about the solute as an empty layer devoid of watersurrounding the solute sphere. Border thicknesses for small solutestypically range from $0.4 \AA$ to $0.6 \AA$, although values up to $1.0 \AA$ has been observed forproteins[4]. Recent simulationsperformed by Chalikian and coworkers[2,5] of a series of solutes composed of atomic Lennard-Jones (LJ) sites with their partial charges turnedoff to make them nonpolar found that the spherical border thickness grew with increasing solute size. This observation was interpreted as water pulling away from the solute and dewetting the solute/solvent interface as predicted by scaled-particle[6-8] and Lum-Chandler-Weeks[9]theories. Here we analyze this surprising, and potentially important, result by comparing the measured border thicknesses against simulations of spherical solutes of varying size in water and a simple geometric model to account, in part, for the solute shape.

Figure 1 compares the border thicknesses of repulsive, spherical solutes (modeled as shifted Weeks-Chandler-Andersen (WCA) particles[10]) evaluated from simulations in TIP4P/2005 water [11]at $25^{\circ} \mathrm{C}$ and $1 \mathrm{~atm}$ (see Methods) as a function of their sizeagainst those determined by Chalikian and co-workers[2, 5]for molecularly detailed non-polar solutes (Table 1). For the repulsive spheres we find that the border thickness is an increasing function of the solute size, consistent with solute dewetting[12]. Dewetting is marked by depression of the water density in the first hydration shell with increasing solute size[6-9] resulting from an imbalance of forces for solvent molecules partially stripped of their full solvation shell next to a repulsive, low 
curvature interface[13, 14].It has previously been demonstrated in simulations of hard-sphere solutes in water [12] and Lennard-Jonesium[8] that depression of the solvent density next to solutes larger than the solvent is accompanied by net solvent desorption. This solvent desorption in turn is directly tied to excess growth in the solute's partial molar volume greater than would be anticipated with the change of the solute's van der Waals radius [8]. The spherical border thickness of the molecular solutes with effective radii up to $\sim 4 \AA$ is approximately constant with $\delta_{\mathrm{sph}} \approx 0.5 \AA$, in agreement with experimental correlations for the border thickness (Figure 1). The border thickness is an increasing function of size for molecular solutes with effective radii greater than $\sim 4 \AA$, however, falling just below that of similarly sized repulsive spheres for the largest solutes simulated here.

A shortcoming of the comparison made above is that we neglected attractive solute/water interactions while dispersive interactions were included in the molecular solute simulations composed of LJ sites. The attractive volume increment can be evaluated by a series expansion about the repulsive solute reference system[15]. Truncating this expansion after the first order term, the attractive contribution to the volume is given by the integral

$$
\Delta V_{\mathrm{att}} \approx(R T)^{-1} \int \varphi_{\mathrm{att}}\left(r-r_{\text {shift }}\right) \chi(r) 4 \pi r^{2} d r,
$$

where $\varphi_{\text {att }}\left(r-r_{\text {shift }}\right)$ is the shifted WCA attractive interaction (see Methods), while the solute volume's interaction susceptibility, $\chi(r)$, is determined by the cross fluctuation between the system volume and water density as a function of distance from the solute, i.e., $\chi(r)=$ $\langle V\rangle\left\langle\rho_{\mathrm{sw}}(r)\right\rangle-\left\langle V \rho_{\mathrm{sw}}(r)\right\rangle[15]$. The interaction susceptibilities for three solutes of increasing size determined from the repulsive spheres in water are reported in Figure 2. Thisplot shows the susceptibility is largest for waters in the first hydration shell of the solute. This growth of the 
susceptibility in the hydration shell results from the increasing compressibility of the dewetting layer making the proximal water density increasingly sensitive to attractive perturbations[16].

The border thicknesses of the spherical solutes with $\Delta V_{\text {att }}$ added to the repulsive solute volumes drop significantly (Figure 1), indicating attractions counter dewetting and draw water backtowards the solute. The comparison between $\delta_{\mathrm{sph}}$ for the attractive spheres and molecular solutes with radii less than $4 \AA$ is excellent. For solutes larger than $4 \AA$, however, $\delta_{\text {sph }}$ for the molecular solutes depart from those of theattractive spheres and begin to rise towards that of the repulsive spheres. This suggests that water gradually pulls away from the molecular LJ solutes beyond a critical solute size, despite the fact that attractions draw water closer to spherical solutesfor all sizes.

The inference that molecular nonpolar solutes dewet is surprising and stands in difference to successfulpartial molar volume group contribution correlations, which assume the solute's hydrated volume is the sum of its constituent group volumes without regard to the number of groups making up the solute[17-19].Indeed, the group volumes largely depend only on local correlations between the group and neighboring water molecules[20]. The growth of the border thickness, however, rests on the assumption that molecular solutes can be approximated as a sphere. Expanding eq. (1) to its leading order in $\delta_{\mathrm{sph}}$, i.e., $V_{\mathrm{hyd}} \approx 4 \pi r_{\mathrm{vdw}}^{3} / 3+4 \pi r_{\mathrm{vdW}}^{2} \delta_{\text {sph }}+$ ..., the hydrated volume is dominated by the solute's van der Waals volume plus a solvent contribution proportional the sphere's area. Since spheres exhibit the smallest area for a given volume it may be anticipated that eq. (1)will breakdown for molecularly detailed solutes, which necessarily have larger exposed surface areas. Moreover, since the surface area-to-volume ratio is a decreasing function of a sphere's radius we may expect unphysical growth of the calculated border thickness with increasing solute size. Indeed alternate volume correlations utilizing the 
phenomenological border thickness apply it to the solute's actual van der Waals area. A more realistic correlation for the hydrated solute volume may be obtained by considering the solute's molecular topology explicitly tocalculate the border thickness. In this case we evaluate the hydrated volume by adding the border thickness (referred to below as $\delta_{\text {mol }}$ ) to the van der Waals radii of the constituent atoms that make up the solute (Methods). Figure 3 and Table 1 compare $\delta_{\mathrm{sph}}$ and $\delta_{\mathrm{mol}}$ as a function of the soluteeffective van der Waals radii. In difference to $\delta_{\mathrm{sph}}, \delta_{\mathrm{mol}}$ is well behaved over the range of sizes considered, adopting values between $0.32 \AA$ and $0.55 \AA$ that are more in line with expectations. The question remains, why does $\delta_{\text {sph }}$ grow unexpectedlywith increasing molecular solute size?

A simple model to illustrate how a solute's border thickness can be significantly overestimated is to consider the geometry of spherocylinders. The van der Waals volume of a spherocylinder with a molecular radius $R$ and end cap-to-cap length $L$ is

$$
V_{\mathrm{vdW}}^{\mathrm{cyl}}=4 \pi R^{3} / 3+\pi R^{2} L
$$

while it's hydrated volumefor a fixed border thickness $\delta_{\text {mol }}$ is

$$
V_{\mathrm{hyd}}^{\mathrm{cyl}}=4 \pi\left(R+\delta_{\mathrm{mol}}\right)^{3} / 3+\pi\left(R+\delta_{\mathrm{mol}}\right)^{2} L .
$$

The effective spherical border thickness of the spherocylinder subsequently is $\delta_{\mathrm{sph}}=$ $\left(3 V_{\mathrm{hyd}}^{\mathrm{cyl}} / 4 \pi\right)^{1 / 3}-\left(3 V_{\mathrm{vdW}}^{\mathrm{cyl}} / 4 \pi\right)^{1 / 3}$. We plot $\delta_{\mathrm{sph}}$ for spherocylinders as a function of $r_{\mathrm{vdW}}^{\mathrm{eff}}(=$ $\left.\left(3 V_{\mathrm{vdW}}^{\mathrm{cyl}} / 4 \pi\right)^{1 / 3}\right)$ in Figure 3 assuming $R=1.9 \AA$, comparable to the effective van der Waals radius of methane, and $\delta_{\mathrm{mol}}=0.44 \AA$, which falls in the middle of the values reported in Table 1, while allowing $L$ to vary from zero on up.The spherical border thickness for spherocylinders grows by more than a factor of two over than range of solute sizes plotted. More interestingly, the spherocylinder modelpredictions closely follow the $\delta_{\mathrm{sph}}$ 's for the molecular solutes reported 
in Table 1. Clearly the growth of $\delta_{\mathrm{sph}}$ for the spherocylinder model is not a result of dewetting since $\delta_{\mathrm{mol}}$ is held fixed in this calculation. Rather this indicates a breakdown in the pseudospherical description of the solute volume. Indeed, the spherocylinder model predicts $\delta_{\text {sph }}$ grows in proportion to $r_{\mathrm{vdW}}^{\mathrm{eff}}$ and does not plateau as might be anticipated for dewetting of a large spherical nonpolar surface[2, 8].

The accuracy of the spherocylinder model should not be taken as validation that these solutes are cylindrical, which is geometrically unreasonable given the range of molecular structures reported in Table 1 and simplicity of the description embodied by eq. [3]. Instead we believe the spherocylinder model'ssuccess results from the qualitatively improved balance between growth of the solute van der Waals area relative to its volume as captured by the ratio $A_{\mathrm{vdW}} / V_{\mathrm{vdW}}$. The area-to-volume ratio for the molecular solutes is a weakly decreasing function of the solute size (Figure 4 with $A_{\mathrm{vdW}}$ reported in Table 1), appearing to plateau for large solutes. For a sphere the ratio $A_{\mathrm{vdW}} / V_{\mathrm{vdW}}=3 / r_{\mathrm{vdW}}$ falls well below that over the molecular solutes,dropping to zero in the limit of an infinitesphere (Figure 4). For a spherocylinder, with a van der Waals area of $A_{\mathrm{vdW}}^{\mathrm{cyl}}=4 \pi R^{2}+2 \pi R L$, the area to volume ratioonly falls slightly with increasing solute size and plateaus at $1.05 \AA^{-1}(=2 / R$ with $R=1.9 \AA)$ in the infinite solute limit (Figure 4).While $A_{\mathrm{vdW}} / V_{\mathrm{vdW}}$ for molecular solutes is typically greater than that for similarly sized spherocylinders, the difference is small compared to that for the sphere and the predicted plateau is reasonable. While the spherocylinder model underestimates the molecular solute van der Waals area for a given solute size, the fact that the area-to-volume ratio for both the molecular and spherocylinder descriptions plateau over a similar size scale indicates that the differences can be accommodated by the spherocylinder model by utilizing a slightly larger 
empirical values of $\delta_{\mathrm{mol}}$. The spherical model for the solute volume, on the other hand, would be unable to overcome its deficiencies with a simple constant border thickness.

In summary, while purely repulsive spherical solutes in water do show indications of dewetting with increasing solute radius manifest as solute/solvent border thickening, attractive interactions suppress dewetting. The apparent dewetting of molecularly detailed nonpolar solutes with increasing size, however, is not real. Rather, the growth of the phenomenological spherical border thickness results from a breakdown of the spherical solute approximation applied to molecular solutes. If the solute's molecular structure is taken into account when evaluating the hydrated solute volume the border thickness varies significantly less from solute to solute with values ranging from $0.32 \AA$ to $0.55 \AA$. Beyond the van der Waals volume then, our observations demonstrate that an improved accounting of water packing against the solute's area improves the phenomenological description of the hydrated volume.

\section{Methods}

Simulations ofindividual repulsive, spherical solutes in aqueous solution were performed in the isothermal-isobarric ensemble using the GROMACS 5.0 simulation package[21]. The temperature and pressure were fixed at $25^{\circ} \mathrm{C}$ and 1 bar using the Nosé-Hoover thermostat[22, 23] and Parrinello-Rahman barostat[24], respectively. Water was modeled using the TIP4P/2005 model[11], while solute/water interactions for the repulsive spherical solute was determined by the shifted WCA potential[10], $\varphi_{\mathrm{WCA}}\left(r-r_{\mathrm{shift}}\right)$, where the position at which the solute/water interaction diverges to infinity has been shifted from the origin to $r_{\text {shift }}$. The WCA potential represents the repulsive portion of the shifted LJpotential following the standard WCA perturbation theory divisionbetween attractive and repulsive forces, i.e., $\varphi_{\mathrm{LJ}}\left(r-r_{\text {shift }}\right)=$ 
$\varphi_{\mathrm{WCA}}\left(r-r_{\text {shift }}\right)+\varphi_{\text {att }}\left(r-r_{\text {shift }}\right)$. The LJ well-depth and diameter used here are $3.444 \AA$ and $0.9765 \mathrm{~kJ} / \mathrm{mole}$, respectively, corresponding to the cross interactions between water and methane modeled as a united-atom site[25] obtained using Lorentz-Berthelot combining rules[26]. Simulated values of $r_{\text {shift }}$ ran from $0 \AA$ to $7 \AA$ in $0.5 \AA$ increments. Simulations were conducted of one solute in a bath of anywhere between 648 and 2307 water molecules depending on the solute size. An additional simulation of 1000 water molecules and no added solute was performed to characterize pure solvent properties. Short range van der Waals interactions were truncated beyond $12 \AA$ with no long-range correction, while electrostatic interactions were evaluated using particle-mesh Ewald summation[27]. Water's internal constraints were held fixed using the SETTLE algorithm[28]. Simulations were conducted for $100 \mathrm{~ns}$ following at least $1 \mathrm{~ns}$ for equilibration using a time step of 2 fs. Partial molar volumes for the solutes were determined as the difference in the average simulation volume for the system with and without the solute added, i.e., $\bar{V}_{\text {solute }}=\langle V\rangle_{\text {water+solute }}-\langle V\rangle_{\text {water }}$ with the pure water simulation volume scaled to match the number of waters in the mixture. The ideal gas contribution to the partial molar volume was determined from pure solvent simulation fluctuations to be $R T \kappa_{0}=2.02 \AA^{3}$.

The van der Waals radius of the shifted WCA solutes can be determined from their effective solvent excluded volume. This volume is determined here by the integral

$$
\left(r_{\mathrm{vdW}}+\sigma_{\mathrm{wat}} / 2\right)^{3}=3 \int_{0}^{\infty}\left\{1-\exp \left[-\varphi_{\mathrm{WCA}}\left(r-r_{\mathrm{shift}}\right) / R T\right]\right\} r^{2} d r
$$

where $\sigma_{\text {wat }}=2.8 \AA$ is the effective hard-sphere diameter of water.

The van der Waals areas and volumes reported in Table 1 were determined using Monte Carlo integration. The atomic van der Waals radii of the carbon, hydrogen, nitrogen, and oxygen atoms used were $1.7 \AA, 1.2 \AA, 1.55 \AA$, and $1.55 \AA$. To determine the volume of each molecule they were placed in a bounding box and choosing $2.5 \times 10^{8}$ random points. The molecular 
volume (either van der Waals or hydrated volume) was determined as the volume of the box times the number of points found to lie within a specified radius (either the van der Waals radius of each atom or the van der Waals radius plus the molecular border thickness $\delta_{\text {mol }}$ ) of any atomic site of the molecule divided by the number of points inserted into the box. The molecular border thicknesses reported in Table 1 were obtained by fitting the volumes obtained by Monte Carlo integration to the hydrated volumes reported by Chalikian and coworkers by adjusting $\delta_{\text {mol }}$. The error in the volumes was found to be $\pm 0.02 \AA^{3}$ or less. To determine the van der Waals surface area of each molecule, $2.5 \times 10^{8}$ random points where chosen on thevan der Waals sphere about each atom of the molecule. The van der Waals area of an atom was determined as the fraction of random points found to be outside the van der Waals radii of all the other atoms of the molecule times the area of the sphere with the van der Waals radius of that atom. The total van der Waals area was subsequently determined as the sum of a molecule's constituent atomic van der Waals areas.The error in the areas was found to be $\pm 0.02 \AA^{3}$ or less.

\section{Acknowledgements}

We gratefully acknowledge financial support from a Louisiana Board of Regents Graduate Research Fellowship (JWB), the NSF (OIA-1430280), and Tulane's Center for Engaged Learning and Teaching. 


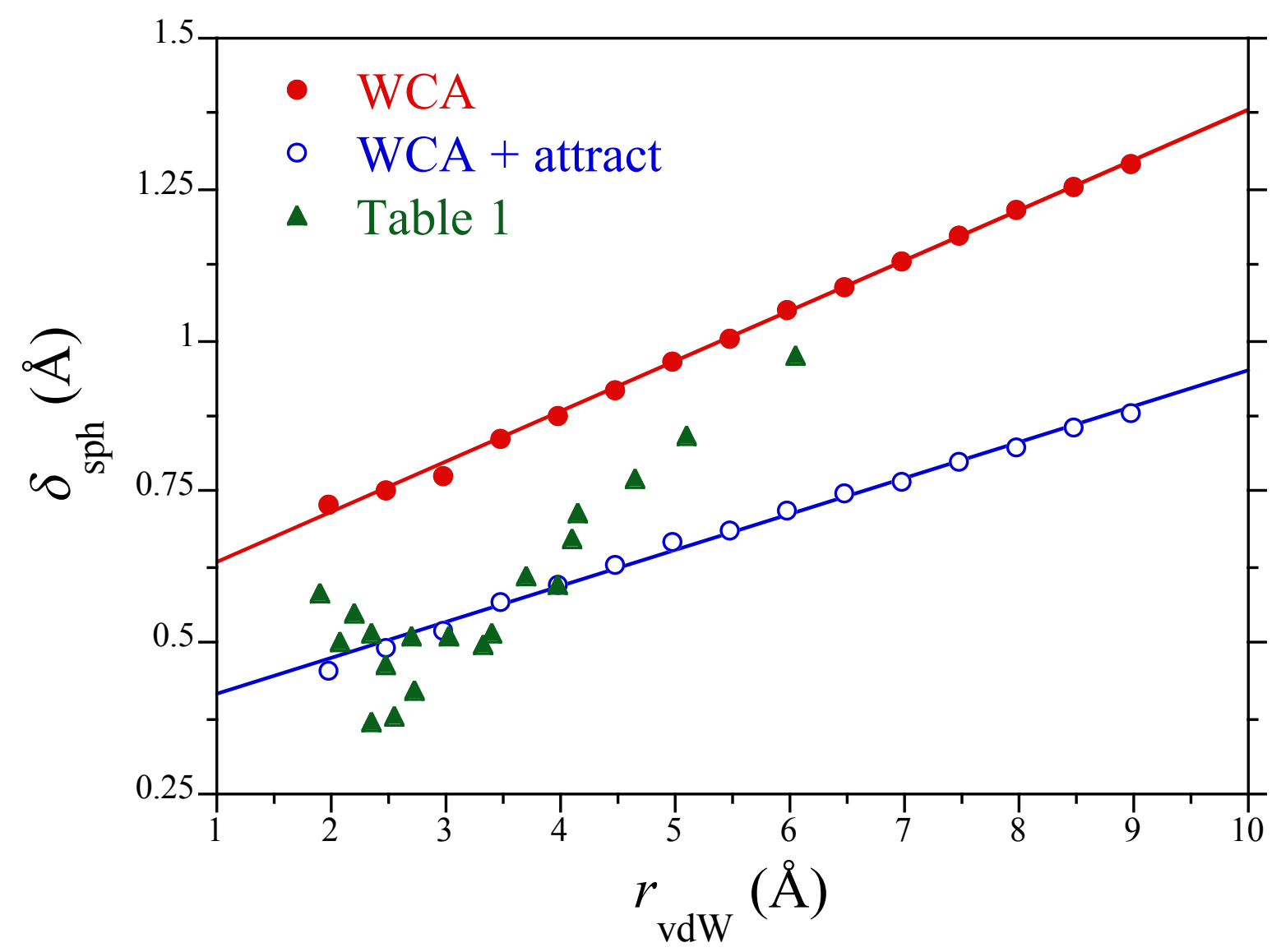

Figure 1.Dependence of the volumetric border thickness on the effective van der Waals radius. Results are reported for shifted WCA solutes in water without and with (via eq. [1]) added attractions as well as for the molecular solute simulation results reported in Table 1. Symbols are defined in the figure legend. The straight lines through the WCA simulation results serve only as a guide for the eye. 


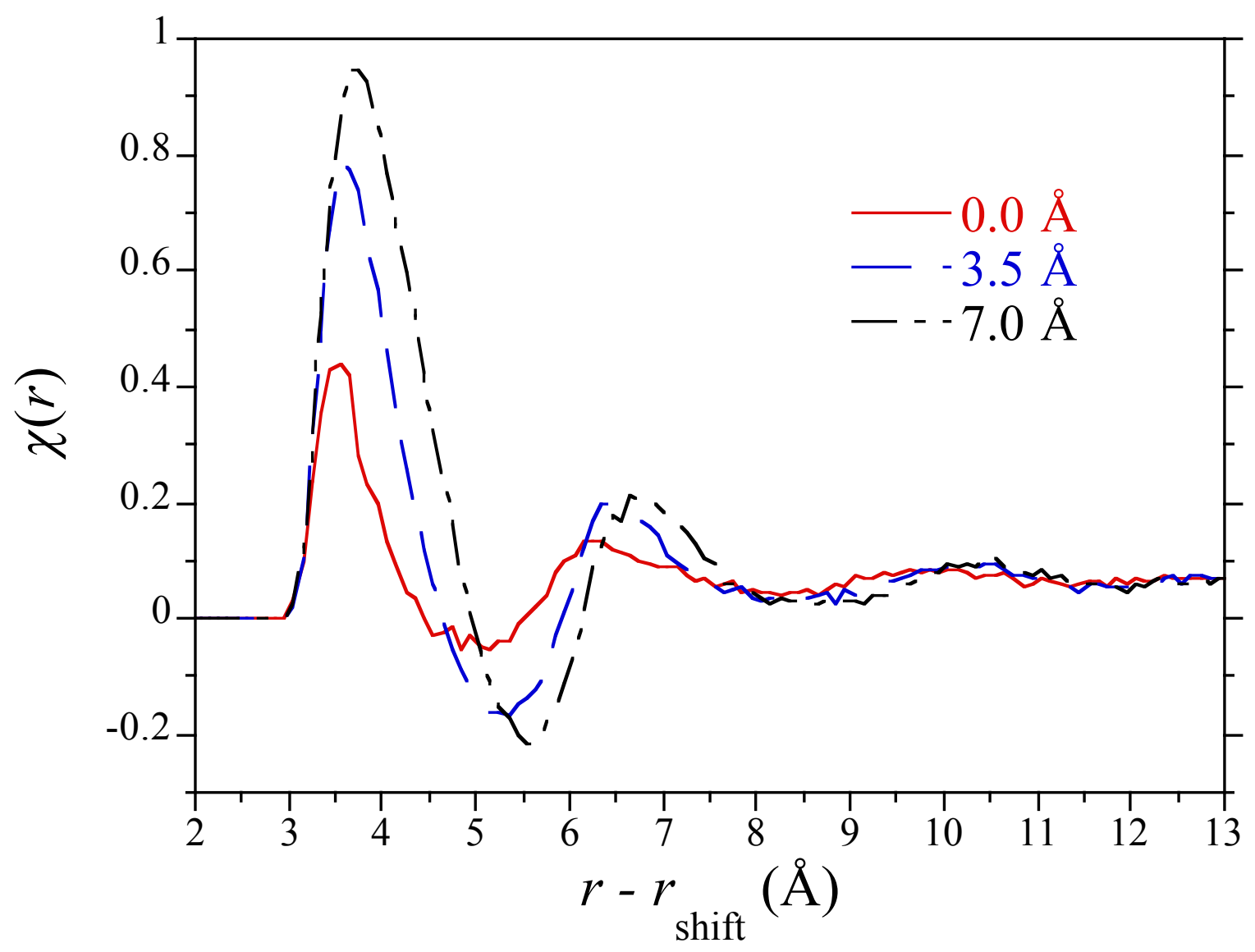

Figure 2. Shifted WCA solute volume attraction response function determined from molecular simulations. Results are reported for three differentWCA shifts, with the $x$-axis shifted by $r_{\text {shift }}$ to facilitate comparison. Lines indicating different values of $r_{\text {shift }}$ are defined in the figure legend. 


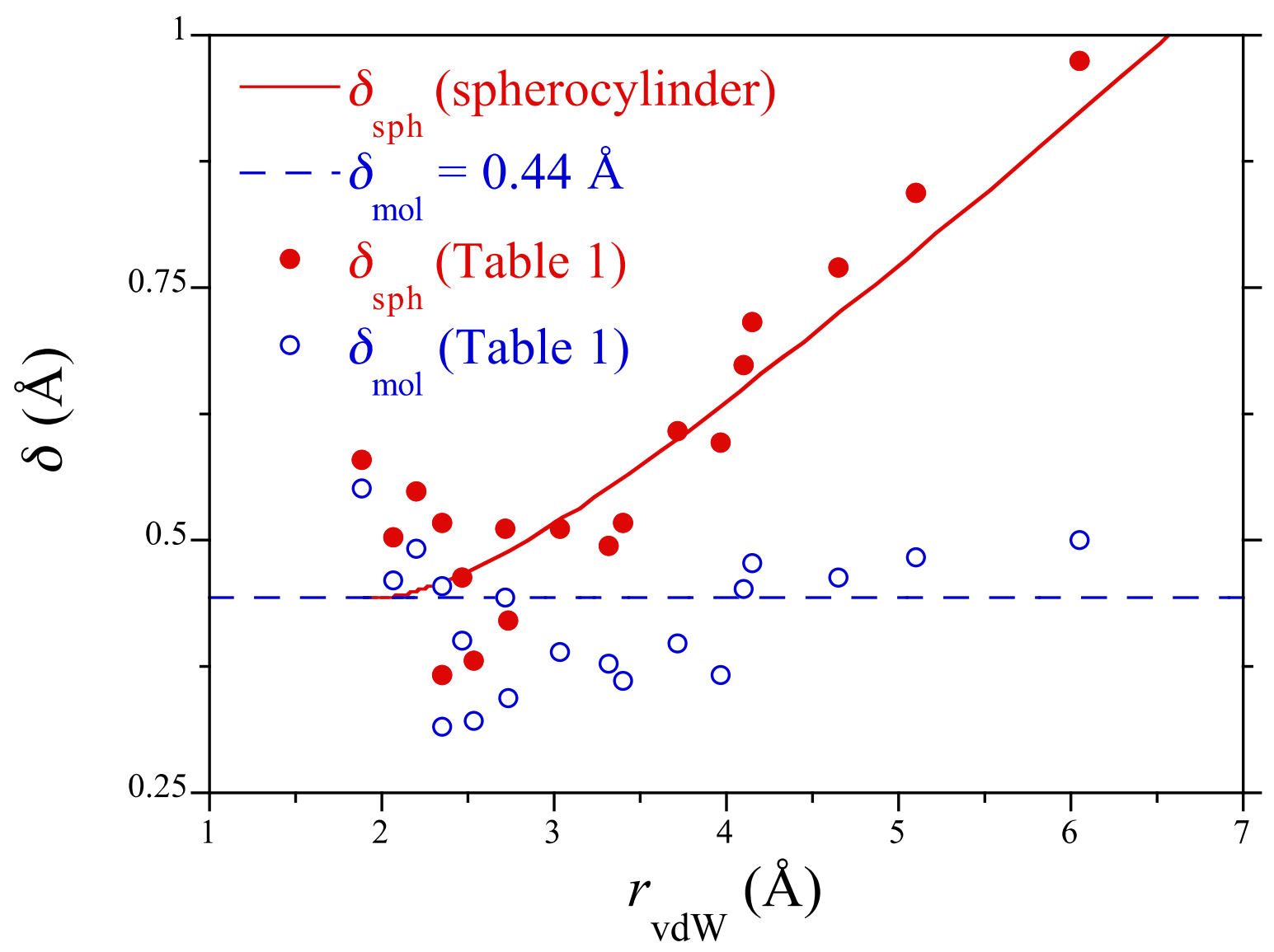

Figure 3. Comparison between the effective border thicknesses determined based on the spherical versus molecular approximations as a function for the van der Waals radius for the solutes reported in Table 1. 


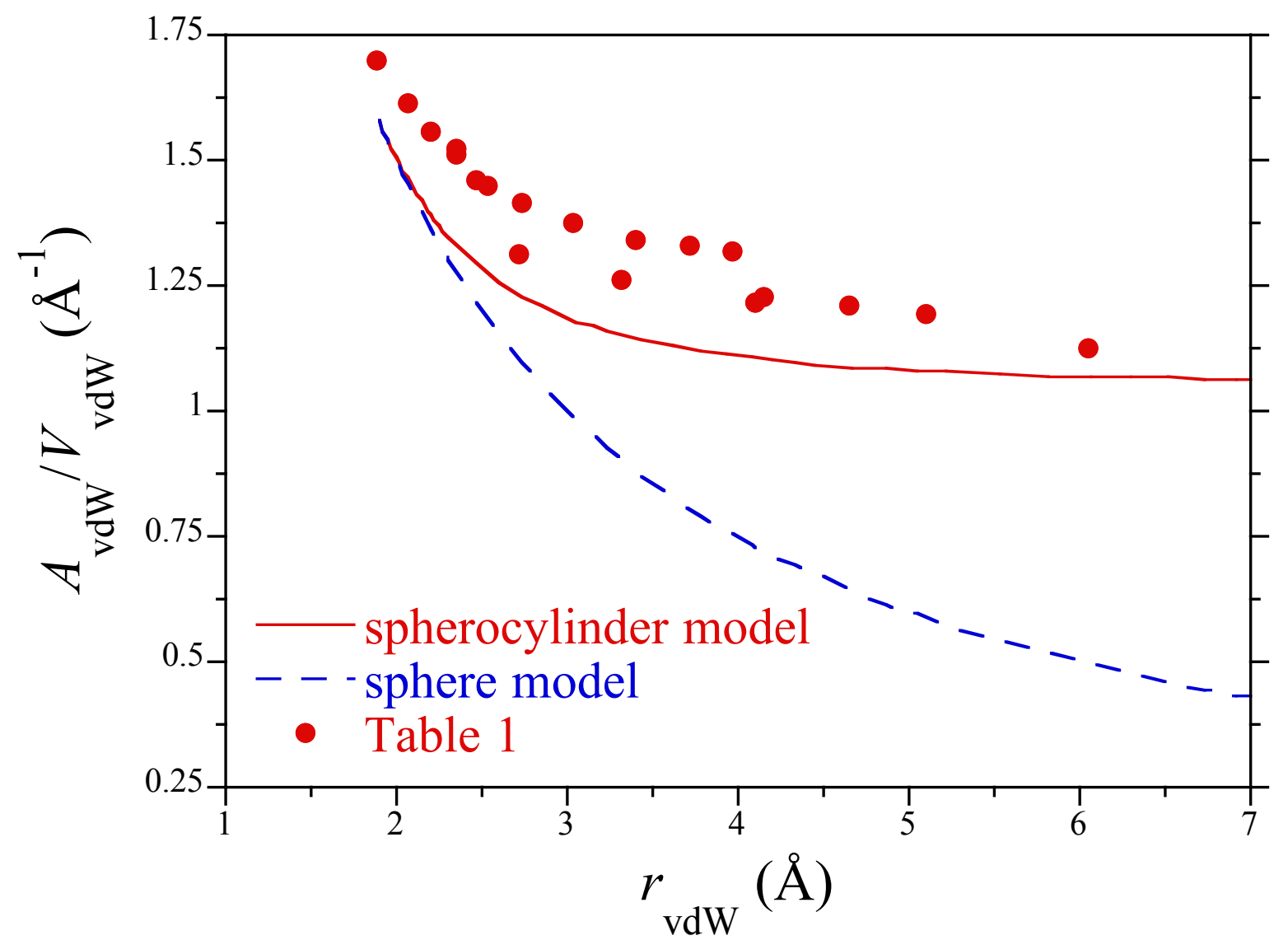

Figure 4. Ratio of the van der Waals area to volume as a function the van der Waals radius. Results are reported for thespherocylinder and spherical models as well as for the solutes reported in Table 1. 

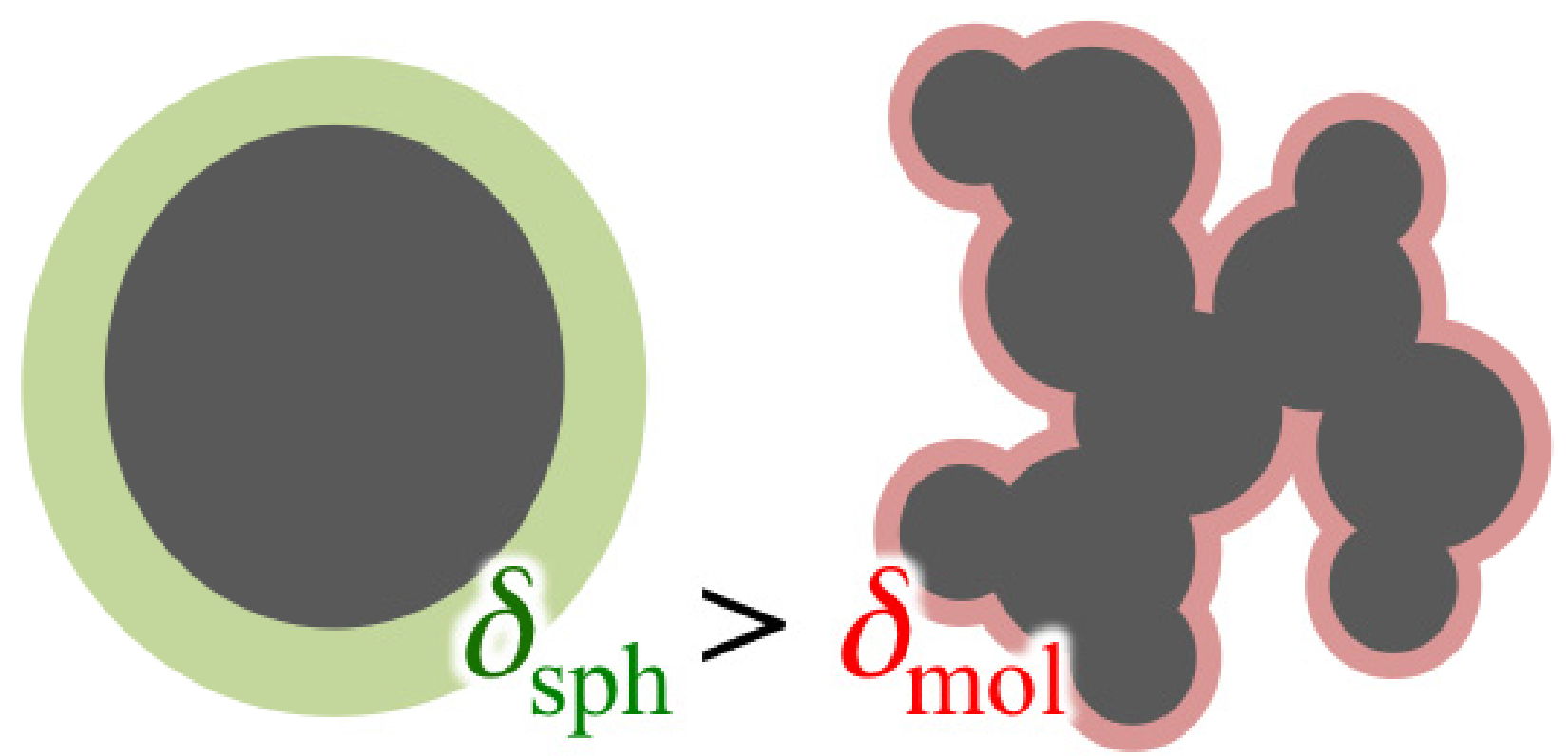

Table of Contents Figure

\section{Highlights}

- The partial molar volumes of molecular nonpolar solutes in water are shown to vary between that of an attractive spherical solute to a repulsive spherical solute with increasing solute size, suggesting systematic dewetting of the molecular solutes.

-When the topology of the molecular solute is taken into account, the dewetting inferred from growth of the phenomenological spherical border thickness is found to disappear.

- A simple geometrical model based on spherocylinder geometry is shown to account for the effective growth of spherical border thickness. 
Table 1. Volumetric properties of the nonpolar molecular solutes in TIP3P water at $25^{\circ} \mathrm{C}$ and 1 atm reported by Chalikian and coworkers. All partial charges were turned off for these solutes with only Lennard-Jones interactions between the solute sites and water. The hydration volume was determined from the simulation partial molar volumes as $V_{\text {hyd }}=\bar{V}_{\text {solute }}-R T \kappa_{0}$ (errorsreported in parentheses). The van der Waals volumes and areas were determined by Monte Carlo integrationas described in the Methods section. The spherical border thicknesseswere determined as $\delta_{\mathrm{sph}}=\left(3 V_{\mathrm{hyd}} / 4 \pi\right)^{1 / 3}-\left(3 V_{\mathrm{vdW}} / 4 \pi\right)^{1 / 3}$, while the $\delta_{\mathrm{mol}}$ were fitted to $V_{\mathrm{hyd}}$ by Monte Carlo integrationas described in the Methods section.

$\begin{array}{lccccc}\text { solute } & V_{\mathrm{hyd}}\left(\AA^{3}\right) & V_{\mathrm{vdW}}\left(\AA^{3}\right) & A_{\mathrm{vdW}}\left(\AA^{2}\right) & \delta_{\text {sph }}(\AA) & \delta_{\text {mol }}(\AA) \\ \text { methane } & 63.1(1.2) & 28.3 & 48.0 & 0.58 & 0.55 \\ \text { ethane } & 87.8(1.3) & 45.1 & 70.3 & 0.55 & 0.49 \\ \text { benzene } & 140.3(1.7) & 83.5 & 109.5 & 0.51 & 0.44 \\ \text { methanol } & 71.4(1.5) & 37.2 & 59.9 & 0.50 & 0.46 \\ \text { ethanol } & 98.1(1.0) & 53.9 & 81.5 & 0.52 & 0.45 \\ \text { 1,2-ethanediol } & 105.1(2.8) & 62.7 & 91.7 & 0.46 & 0.40 \\ \text { urea } & 84.0(2.0) & 54.3 & 82.7 & 0.37 & 0.32 \\ \text { alanine } & 131.3(1.5) & 85.4 & 120.7 & 0.42 & 0.34 \\ \text { glycine } & 104.2(1.2) & 68.6 & 99.3 & 0.38 & 0.32 \\ \text { diglycine } & 186.8(1.9) & 117.1 & 160.7 & 0.51 & 0.39 \\ \text { triglycine } & 253.0(1.8) & 165.6 & 222.3 & 0.52 & 0.36 \\ \text { tetraglycine } & 337.7(1.0) & 214.1 & 284.3 & 0.61 & 0.40 \\ \text { pentaglycine } & 399.8(1.8) & 262.7 & 346.1 & 0.60 & 0.37 \\ \text { galactose } & 233.4(1.2) & 153.8 & 194.3 & 0.50 & 0.38 \\ \text { sucrose } & 453.6(1.8) & 287.1 & 349.6 & 0.67 & 0.45 \\ \text { lactitol } & 480.4(1.3) & 297.8 & 365.4 & 0.72 & 0.48 \\ \text { maltotriose } & 668.2(2.2) & 422.2 & 510.9 & 0.77 & 0.46 \\ \text { stachyose } & 879.1(2.5) & 555.2 & 663.2 & 0.84 & 0.48 \\ \beta \text {-cyclodextrin } & 1453.1(1.8) & 927.9 & 1042.6 & 0.98 & 0.50\end{array}$




\section{References}

[1] J.T. Edward, P.G. Farrell, Relation between van der Waals and partial molal volumes of organic molecules in water, Canadian Journal of Chemistry, 53 (1975) 2965-2970.

[2] N. Patel, D.N. Dubins, R. Pomes, T.V. Chalikian, Size dependence of cavity volume: A molecular dynamics study, Biophysical Chemistry, 161 (2012) 46-49.

[3] G. Graziano, On the magnitude of border thickness in the partial molar volume of cavities in water, Chemical Physics Letters, 570 (2013) 46-49.

[4] T.V. Chalikian, M. Totrov, R. Abagyan, K.J. Breslauer, The hydration of globular proteins as derived from volume and compressibility measurements: Cross correlating thermodynamic and structural data, Journal of Molecular Biology, 260 (1996) 588-603. [5] N. Patel, D.N. Dubins, R. Pomes, T.V. Chalikian, Parsing Partial Molar Volumes of Small Molecules: A Molecular Dynamics Study, Journal of Physical Chemistry B, 115 (2011) 48564862.

[6] F.H. Stillinger, Structure in aqueous solutions of nonpolar solutes from the standpoint of scaled-particle theory, Journal of Solution Chemistry, 2 (1973) 141-158.

[7] H.S. Ashbaugh, L.R. Pratt, Colloquium: Scaled particle theory and the length scales of hydrophobicity, Reviews of Modern Physics, 78 (2006) 159-178.

[8] H.S. Ashbaugh, T.M. Truskett, Putting the squeeze on cavities in liquids: Quantifying pressure effects on solvation using simulations and scaled-particle theory, Journal of Chemical Physics, 134 (2011) 10.

[9] K. Lum, D. Chandler, J.D. Weeks, Hydrophobicity at small and large length scales, Journal of Physical Chemistry B, 103 (1999) 4570-4577.

[10] J.D. Weeks, D. Chandler, H.C. Andersen, Role of repulsive forces in determining the equilibrium structure of simple liquids, Journal of Chemical Physics, 54 (1971) 5237-5247.

[11] J.L.F. Abascal, C. Vega, A general purpose model for the condensed phases of water: TIP4P/2005, Journal of Chemical Physics, 123 (2005) 234505.

[12] F.M. Floris, Nonideal effects on the excess volume from small to large cavities in TIP4P water, Journal of Physical Chemistry B, 108 (2004) 16244-16249.

[13] J.D. Weeks, K. Katsov, K. Vollmayr, Roles of repulsive and attractive forces in determining the structure of nonuniform liquids: Generalized mean field theory, Physical Review Letters, 81 (1998) 4400-4403.

[14] G. Hummer, S. Garde, Cavity expulsion and weak dewetting of hydrophobic solutes in water, Physical Review Letters, 80 (1998) 4193-4196.

[15] S.M. Williams, H.S. Ashbaugh, Note: Nonpolar solute partial molar volume response to attractive interactions with water, Journal of Chemical Physics, 140 (2014) 2.

[16] M.V. Athawale, S.N. Jamadagni, S. Garde, How hydrophobic hydration responds to solute size and attractions: Theory and simulations, Journal of Chemical Physics, 131 (2009) 9.

[17] A.V. Plyasunov, E.L. Shock, Group contribution values of the infinite dilution thermodynamic functions of hydration for aliphatic noncyclic hydrocarbons, alcohols, and ketones at 298.15 K and 0.1 MPa, Journal of Chemical and Engineering Data, 46 (2001) 1016-1019.

[18] G.R. Hedwig, H.J. Hinz, Group additivity schemes for the calculation of the partial molar heat capacities and volumes of unfolded proteins in aqueous solution, Biophysical Chemistry, 100 (2003) 239-260. 
[19] L.N. Surampudi, H.S. Ashbaugh, Direct Evaluation of Polypeptide Partial Molar Volumes in Water Using Molecular Dynamics Simulations, Journal of Chemical and Engineering Data, 59 (2014) 3130-3135.

[20] A.V. Sangwai, H.S. Ashbaugh, Aqueous partial molar volumes from simulation, and individual group contributions, Industrial \& Engineering Chemistry Research, 47 (2008) 5169-5174.

[21] B. Hess, C. Kutzner, D. van der Spoel, E. Lindahl, GROMACS 4: Algorithms for Highly Efficient, Load-Balanced, and Scalable Molecular Simulation, Journal of Chemical Theory and Computation, 4 (2008) 435-447.

[22] S. Nosé, A Unified Formulation of the Constant Temperature Molecular-Dynamics Methods, Journal of Chemical Physics, 81 (1984) 511-519.

[23] W.G. Hoover, Canonical Dynamics: Equilibrium Phase-Space Distributions, Physical Review A, 31 (1985) 1695-1697.

[24] M. Parrinello, A. Rahman, Polymophic Transitions in Single-Crystals - A New Molecular-Dynamics Method, J. Appl. Phys., 52 (1981) 7182-7190.

[25] M.G. Martin, J.I. Siepmann, Transferable potentials for phase equilibria. 1. United-atom description of n-alkanes, Journal of Physical Chemistry B, 102 (1998) 2569-2577.

[26] M.P. Allen, D.J. Tildesley, Computer simulation of liquids, Oxford University Press, Oxford, UK, 1987.

[27] T. Darden, D. York, L. Pedersen, Particle Mesh Ewald: An N·log(N) Method for Ewald Sums in Large Systems, Journal of Chemical Physics, 98 (1993) 10089-10092.

[28] J.P. Ryckaert, G. Ciccotti, H.J.C. Berendsen, Numerical-Integration of Cartesian Equations of Motion os a System with Constraints - Molecular Dynamics of n-Alkanes, Journal of Computational Physics, 23 (1977) 327-341. 